\title{
Harmonization of European Laboratory Response Networks by IMPLEMENTING CWA 15793: Use of a Gap Analysis and an "Insider" Exercise as Tools
}

Bo Sundqvist, Ulrika Allard Bengtsson, Henk J. Wisselink, Ben P. H. Peeters, Bart van Rotterdam, Evelien Kampert, Sándor Bereczky, N. G. Johan Olsson, Åsa Szekely Björndal, Sylvie Zini, Sébastien Allix, and Rickard Knutsson

Laboratory response networks (LRNs) have been established for security reasons in several countries including the Netherlands, France, and Sweden. LRNs function in these countries as a preparedness measure for a coordinated diagnostic response capability in case of a bioterrorism incident or other biocrimes. Generally, these LRNs are organized on a national level. The EU project AniBioThreat has identified the need for an integrated European LRN to strengthen preparedness against animal bioterrorism. One task of the AniBioThreat project is to suggest a plan to implement laboratory biorisk management CWA 15793:2011 (CWA 15793), a management system built on the principle of continual improvement through the Plan-Do-Check-Act (PDCA) cycle. The implementation of CWA 15793 can facilitate trust and credibility in a future European LRN and is an assurance that the work done at the laboratories is performed in a structured way with continuous improvements. As a first step, a gap analysis was performed to establish the current compliance status of biosafety and laboratory biosecurity management with CWA 15793 in 5 AniBioThreat partner institutes in France (ANSES), the Netherlands (CVI and RIVM), and Sweden (SMI and SVA). All 5 partners are national and/or international laboratory reference institutes in the field of public or animal health and possess highcontainment laboratories and animal facilities. The gap analysis showed that the participating institutes already have robust biorisk management programs in place, but several gaps were identified that need to be addressed. Despite differences between the participating institutes in their compliance status, these variations are not significant. Biorisk management exercises also have been identified as a useful tool to control compliance status and thereby implementation

Bo Sundqvist, PhD, is QA Project Leader, Department of Security; Rickard Knutsson, PhD, is Director, Department of Security; and Ulrika Allard Bengtsson, MSc Agr, is Biosafety Officer and Research Scientist, Department of Virology, Immunobiology and Parasitology; all at the National Veterinary Institute (SVA), Uppsala, Sweden. Henk J. Wisselink, Dr, is Biosafety Officer and Research Scientist, Department of Infection Biology, and Ben P. H. Peeters, PhD, is Project Leader, Department of Virology, both at the Central Veterinary Institute of Wageningen UR (CVI), Lelystad, the Netherlands. Bart van Rotterdam, PhD, is Microbiologist, specialized in bioterrorism and Q fever, and Evelien Kampert is Biosafety Officer BSL3/4, both at the National Institute of Public Health and the Environment (RIVM), Bilthoven, the Netherlands. Sándor Bereczky, PhD, is Molecular Biologist; N. G. Johan Olsson, DVM, is Biopreparedness Investigator; and Åsa Szekely Björndal, PhD, is Head of Biorisk Management Unit; all in the Department of Analysis and Prevention, Swedish Institute for Communicable Disease Control (SMI), Solna, Sweden. Sylvie Zini, PharmD, PhD, is Defence Representative and Biosafety and Biosecurity Coordinator, General Directorate, and Sébastien Allix, is Biosafety Officer, Department of Maisons-Alfort Laboratory for Animal Health, both at the French Agency for Food, Environmental and Occupational Health \& Safety (ANSES), Maisons-Alfort, France. 
of CWA 15793. An exercise concerning an insider threat and loss of a biological agent was performed at SVA in the AniBioThreat project to evaluate implementation of the contingency plans and as an activity in the implementation process of CWA 15793. The outcome of the exercise was perceived as very useful, and improvements to enhance biorisk preparedness were identified. Gap analyses and exercises are important, useful activities to facilitate implementation of CWA 15793. The PDCA cycle will enforce a structured way to work, with continual improvements concerning biorisk management activities. Based on the activities in the AniBioThreat project, the following requirements are suggested to promote implementation: support from the top management of the organizations, knowledge about CWA 15793, a compliance audit checklist and gap analysis, training and exercises, networking in LRNs and other networks, and interinstitutional audits. Implementation of CWA 15793 at each institute would strengthen the European animal bioterrorism response capabilities by establishing a well-prepared LRN.

A NIMAL AND ZOONOTIC INFECTIOUS DISEASES are a continuing threat to humans and livestock and have an enormous impact on public health and agriculture as well as on the economy. The misuse of agents and toxins associated with these diseases for terrorism purposes is a real threat.

\section{EU CBRN Action Plan}

The EU Council adopted conclusions on strengthening chemical, biological, radiological, and nuclear (CBRN) security in the European Union and approved an EU CBRN Action Plan. ${ }^{1}$ The EU CBRN Action Plan aims at reducing the threat and possible consequences of CBRN incidents of natural, accidental, or intentional origin, including acts of terrorism. It contains a large number of actions concerning prevention, detection, preparedness, and response, as well as horizontal measures in the context of high-risk CBRN materials. As stated, "The EU CBRN Action Plan is broadly based on an all-hazard approach, including terrorist threats, and contributes to the implementation of the EU Counter Terrorism Strategy. The Action Plan constitutes a political commitment, which may be seen as a roadmap of intentions for the coming years." $1(\mathrm{p} 9)$

\section{CWA 15793}

The EU CBRN Action Plan has identified the need for different kinds of activities that increase awareness and understanding of and conformity with biosafety and laboratory biosecurity. In this article, we use the term "biosecurity" to mean laboratory biosecurity. One of the identified activities is the use of a management system and performance measurement tool as an integrated approach to laboratory biosafety and laboratory biosecurity, collectively referred as "biorisk" (Table 1).

In the Action Plan (B.4), this is referred to as the implementation of CEN Workshop Agreement 15793:2011 "Laboratory biorisk management" (CWA 15793). ${ }^{2}$ CWA
15793 was initiated and adopted because organizations, laboratories, and others involved in biosafety and biosecurity activities identified the need for harmonization and conformity regarding biosafety recommendations and guidelines. ${ }^{3-6}$ CWA 15793 is a management system that covers the administrative and operational activities of a facility concerning biorisk and is compatible with other quality management systems (eg, ISO 9001 Quality, ${ }^{7}$ ISO14001 Environment, ${ }^{8}$ ISO18001 Occupational health and safety $\left.{ }^{9}\right)$; it is built around the Plan-Do-Check-Act principle (PDCA cycle) (Figure 1). The key components of CWA 15793 are biorisk assessment (identification of all possible hazards/threats), mitigation measures (procedures to effectively reduce risks), and performance (check whether goals and standards are met). ${ }^{2}$

One way of getting information about the current situation of preparing to start the implementation process of CWA 15793 is to perform a compliance gap analysis of all the topics covered in CWA 15793. Another tool that CWA 15793 has identified as important is the use of exercises to control that routines, plans, and instructions are actually implemented. In CWA 15793 a list of emergency scenarios is included that exemplify various biosafety and laboratory biosecurity risks; several levels of exercises are suggested for the emergency scenarios. ${ }^{2}$

\section{Laboratory Response Networks}

Diagnostic network capabilities must be established for a robust response to a covert or overt bioterrorism incident. A diagnostic response strategy must be able to handle both types of incidents and thus requires a multidisciplinary network composed of diagnostic capabilities in both law enforcement and public health agencies, including environmental, agricultural, food, veterinary, and human medicine organizations. As a result of these demands, laboratory response networks (LRNs) have been developed in several countries. The US LRN was established in 1999; its objective is to ensure an effective laboratory response to bioterrorism by improving the law enforcement and public 
Table 1. Biorisk: Relationship Between Laboratory Biosafety and Laboratory Biosecurity with Some Examples of Operational Topics

\begin{tabular}{|lll|}
\hline Laboratory Biosafety & Shared Topics & Laboratory Biosecurity \\
\hline - Hazard identification & - Risk assessment & - Information security \\
- Good laboratory practices and techniques & - Accountability & - Transfer security \\
- Personal protective equipment & - Access restriction and control & - Personal security \\
- Physical containment & - Compliance with standards & - Threat assessment \\
- Personal safety & - Emergency and preparedness routines & - Incident reporting and planning of exercises \\
- Worker health program & - Inventory control & - Inspection and audit \\
& - Standard operating procedures (SOPs) \\
& - Waste management \\
& - Training and education \\
& - Transport \\
& - Management review process \\
& - Personnel reliability \\
\hline
\end{tabular}

health laboratory infrastructure. ${ }^{10}$ The US LRN links local, state, and national public health laboratories, as well as agriculture, veterinary, military, and water- and food-testing laboratories. The US LRN also has collaborations with locations in Canada, Australia, Japan, the United Kingdom, and Germany.

Several other countries have similar laboratory networksfor example, Canada, ${ }^{11}$ Australia, ${ }^{12}$ and South Korea. ${ }^{13}$ The Swedish Laboratory Response Network (Svenska Laboratorieresponsnätverket) ${ }^{14}$ was established in 2009 to facilitate collaboration among law enforcement, first responders, veterinary agencies, and food safety and public health agencies. In the Netherlands in 2005, a National Network of Laboratories for Terrorist Attacks (Landelijk Laboratorium Netwerk terreur aanslagen, LLN-ta) ${ }^{15}$ was formed to have laboratory capacity and expertise available

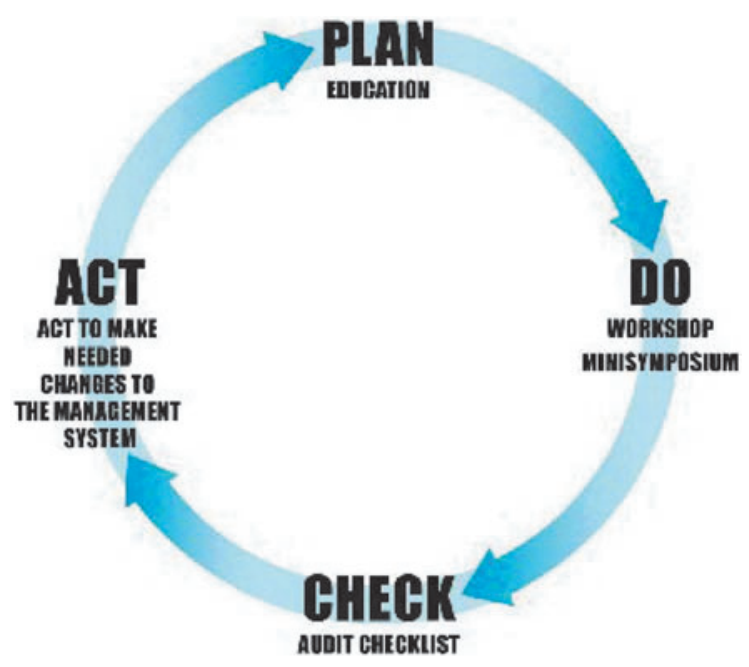

Figure 1. Biorisk Management System. Implementation of the biorisk management system according to the Plan-Do-Check-Act principle (PDCA cycle) in case of CBRN incidents and to enable a quick response to a request for assistance in analyzing unknown samples for a variety of potential agents. ${ }^{16}$ In France, the National Network of Biotox-Piratox laboratories was established in $2001 .^{17}$ It provides a government plan of response to threats and acts of terrorism based on a national network of laboratories. The plan is activated in component Biotox for biological threat, as Piratox for a chemical threat, and as component Piratome for nuclear and radiological threats.

\section{AniBioThreat}

The EU AniBioThreat project ${ }^{18}$ aims to improve the EU's capacity to counter biological animal terrorism threats in terms of awareness, prevention, and contingency planning by building bridges across boundaries dividing countries, competencies, and disciplines. The focus of the project is on threats to living animals, animal feed, and food of animal origin. Furthermore, the project aims to enhance international cooperation and promote multidisciplinary networking for bridging security measures between animal health and public health, the One Health initiative approach. ${ }^{19}$

One of the objectives of the AniBioThreat project is to establish a European LRN approach to countering animal bioterrorism threats. A specific task in this part is to inform and increase the awareness of the concept of biorisk and to plan and initiate implementation of CWA 15793. By implementing the CWA 15793 in the national response networks such as in France, the Netherlands, and Sweden, the harmonization for a future European response network will be facilitated. A gap analysis and exercise has been conducted to start the process.

\section{Gap Analysis and "Insider" Exercise}

As a first step toward implementing CWA 15793, a gap analysis was performed to determine the current

Biosecurity and Bioterrorism: Biodefense Strategy, Practice, and Science 
compliance with CWA 15793 regarding the management of biosafety and laboratory biosecurity in 5 institutes involved in public and veterinary health in France, the Netherlands, and Sweden. Also, an emergency scenario was developed and an "insider" exercise performed at 1 of the partner institutes to evaluate the implementation of emergency and contingency plans. The results of the exercise showed that improvements in the biorisk management system are needed.

\section{Materials and Methods}

\section{Relevant Legislation}

Biorisk management is a multidisciplinary topic in that it involves different actors and legislative frameworks. Many of the laws in this area have been developed on the basis of sector-specific legislation as well as EU legislation. Thus, it is important to be aware of and follow the legislation in the EU and each participating country, such as:

- European directive 2000/54/EC concerning the protection of personnel against the risk of exposure to biological agents at work; ${ }^{20}$

- European directive 2009/41/EC concerning the contained use of genetically modified microorganisms; ${ }^{21}$

- Minimum standards for laboratories working with FMDV in vitro/in vivo of European Commission Decision 2009/869/EC (Appendix 10); ${ }^{22}$ and

- National environmental safety and occupational health acts in which requirements are set for the laboratories and work areas where biological agents are handled.

\section{Participating Institutes and Laboratory Networks}

Five AniBioThreat partner institutes working with agents related to animal and public health in France, the Netherlands, and Sweden participated in this study.

The French agency for Food, Environmental and Occupational Health Safety (ANSES-France) is a public administrative institution reporting to the French ministers for Health, Agriculture, the Environment, Labour, and Consumer Affairs. Its principal mission is to contribute to the protection of human health with respect to the environment, the workplace, and food. It also contributes to animal health and welfare, plant health protection, and the evaluation of the nutritional and functional characteristics of food.

The Central Veterinary Institute of Wageningen UR (CVI) in the Netherlands contributes to the protection of animal and human health through veterinary research. CVI performs legally required research related to notifiable animal diseases for the Dutch government (statutory tasks). CVI is equipped with animal and laboratory facilities de- signed to accommodate the entire range of (animal) experiments under proper containment (up to animal biosafety level 4, ABSL-4).

The National Institute for Public Health and the Environment (RIVM) is the Dutch federal institute that works to prevent and control outbreaks of infectious diseases. RIVM promotes public health and consumer safety and helps to protect the quality of the environment. RIVM uses a wide range of laboratories across the entire biosafety spectrum, up to BSL3, while BSL4 is under construction.

The Swedish Institute for Communicable Disease Control (SMI) is a government authority monitoring the epidemiology and promoting prevention and control of communicable diseases among Swedish citizens. SMI gives expert advice and supports local, regional, and central authorities with operative or political responsibilities for communicable disease control. Preventive disease control is facilitated by diagnostics, research, and methodology development at SMI's containment laboratories (BSL-2, BSL-3, BSL-4, and ABSL-4). As a WHO Collaborating Centre for Biological Safety, SMI provides guidance and training workshops to assist in the implementation of CWA 15793 in national and international laboratories.

The National Veterinary Institute (SVA) is a Swedish national authority that promotes good animal and human health, a healthy environment, and sustainable food production. SVA is an expert organization responsible for diagnostic and prophylactic work and provides expert advice, conducts commissioned investigations and programs for controling contagious diseases, and provides vaccines for both routine and emergency vaccination. SVA has laboratories up to BSL-3 and can handle agents in risk group 3 and epizootic agents. Under the supervision of the Ministry for Rural Affairs, SVA plays a central role in the country in diagnostic services and also as a main partner of an eradication program of infectious diseases.

All 5 laboratories are involved in national LRNs: ANSES in the French National Network of Biotox-Piratox laboratories for biological threats; RIVM and CVI in the Dutch Laboratory Response Network concerning Bioterror (LLN-ta); and SMI and SVA in the Swedish Laboratory Response Network.

\section{Biorisk Compliance Inventory Gap Analysis}

Based on CWA 15793 and CWA 16393 (guidance document to CWA 15793$)^{2,23}$ a gap analysis was performed individually by the 5 institutes. The criteria used were the availability of supporting documents (eg, SOPs, handbooks, guidances) and the level of compliance with these documents. The presence of a supporting document and the level of compliance were scored, depending on how many of the items mentioned in the guidance document were covered in the institute's procedures. The consistency 
of the gap analysis was made as a relative assessment without any attempt to harmonize between institutions. The results from the 5 institutes were combined, and the accumulated result is shown in Table 2 .

The purpose of the gap analysis was to get information about areas that were compliant or not compliant with CWA 15793 and to be able to continue the implementation process. The gap analysis gave a rough estimation of the current situation; further analysis will be needed to get more detailed information.

\section{Biorisk Emergency Scenario and Insider Exercise}

CWA 15793 contains a list of emergency scenarios that exemplify different biosafety and biosecurity issues, such as spill, fire, and missing samples; various levels of exercises are suggested concerning the emergency scenarios. The scenario related to "potential loss of biological agents and toxins through theft or any other reason" (CWA 15793, section 4.4.5.1) was considered to be an important and relevant scenario to assess the strengths and weaknesses of biosecurity and emergency management and its role and work processes in a crisis (CWA 15793, section 4.4.5.2).

A local scenario exercise was carried out at SVA as part of the institute's participation in AniBioThreat. The purpose of the exercise was to identify and analyze the strengths and weaknesses of SVA's safety, security, and emergency management, and its roles and work processes in case an insider was found to be responsible for the loss of a biological agent. Another purpose was to discuss development measures in the short and long term to identify strengths and weaknesses in safety, security, and preparedness management (contingency plans and to evaluate the interagency operability between SVA and the police during crisis management).

The scenario and the exercise were developed in collaboration with 2 other Swedish partners in AniBioThreat, the Swedish Civil Contingencies Agency (MSB) and the Swedish National Police Board (RPS), both of whom took part in the exercise. The exercise involved all of the decision makers in the security and emergency management organization at SVA, including the deputy director-general, security manager, biological agents and feed specialists, communications manager, human resources manager, biosafety officer, state epizoologist, agency lawyer, and head of technical service.

The entire exercise was continuously documented by staff. Exercise observers were present and immediately reported the first findings concerning the exercise development. Additionally, a written questionnaire filled in by the participants and a central evaluation workshop was performed to collect results and experiences from the exercise. This formed the basis for an evaluation report suggesting improvements in the biorisk management system.

\section{REsults}

\section{Gap Analysis Summary}

The combined results of the gap analysis from the 5 institutes demonstrated that the participating institutes already have well-developed biorisk management programs in place. More than three-quarters of the procedures were in place, and the majority of these procedures are in compliance with CWA 15793 (Table 2). The individual results obtained at the institutional level showed that, although there were differences between the institutes in the number of available procedures and their compliance status, none of these institutes differed significantly from the others.

General Requirements and Policy (sections 4.1, 4.2)

The survey indicated that the most important requirements for biosafety management were fulfilled (section 4.1.1) (Table 2). However, the general biorisk management system at the participating institutes was not fully in compliance with the requirements of CWA 15793 because of a lack of coherence and implementation. The documents needed were generally in place, but compliance with those was sometimes not fulfilled. The biorisk management system is currently in use at the participating institutes, with each having its own responsibilities and hierarchy. In certain cases, the lack of a centralized system to establish, maintain, control, communicate, and document biorisk procedures is also an impediment to the process of performance measurement and thus to the PDCA cycle (Figure 1). The survey also showed that some institutes are still missing a specific biorisk management policy, although several biorisk aspects were covered in other policies or the institutes' quality assurance manual.

\section{Planning (section 4.3)}

All 5 institutes have good policies concerning planning for hazard identification, risk assessment, and risk control, and compliance with those policies was also good.

\section{Implementation and Operation (section 4.4)}

At all institutes the biosafety professionals meet regularly, but in some institutes there is no independent review group with a representative cross-section of expertise for biorisk issues to advise management (section 4.4.1.3). New personnel receive appropriate supervision until they have demonstrated competence. However, periodic reexamination of competence is not performed at some institutes (section 4.4.2.2). A communication program including regular consultations, meetings, and briefings for providing information on biorisk management to personnel and other relevant parties needs further compliance in many cases (section 4.4.3). However, in some cases biorisk topics are part of regular meetings where these issues are discussed and communicated.

Work toward a centrally accessible biological and toxin inventory is in progress but is not yet in place at several institutes. All information is, however, available at a

Biosecurity and Bioterrorism: Biodefense Strategy, Practice, and Science 

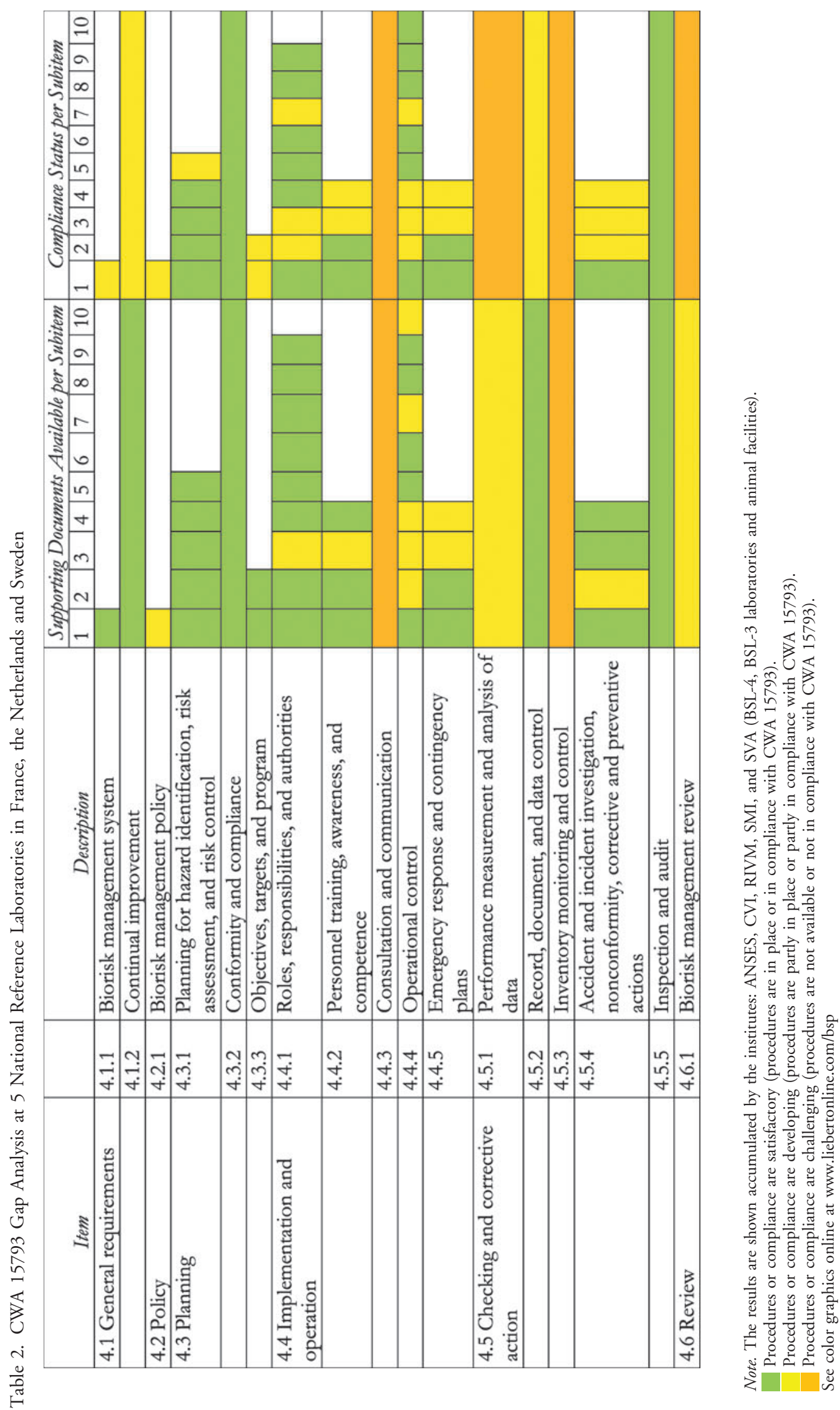
decentralized level (section 4.4.4.2). Other areas that could be improved relate to monitoring of work practices and periodic training programs (section 4.4.4.5). Not all institutes have developed a clear policy regarding personal security support or training. All institutes perform regular emergency exercises in their laboratories and animal facilities for fire alarms and assistance in case of personnel accidents. However, as far as biosafety and biosecurity issues are concerned, there is only limited testing of emergency plans with exercises (section 4.4.5.3). Some institutes lack a comprehensive health monitoring program, but routines for health monitoring of biorisk issues as well as vaccination and other preventive actions according to risk assessment were established (section 4.4.4.6).

\section{Checking and Corrective Action (section 4.5)}

Some institutes do not have a management structure or are in the process of further developing their inventory and control systems for assessing stored biological agents and toxins as well as systems and routines to investigate missing biological agents (section 4.5.3).

Review (section 4.6)

The current management reviews include occupational health biosafety aspects, but generally biosecurity-related items are not included (section 4.6.1).

\section{Insider Exercise}

The AniBioThreat project has developed a number of preparedness scenarios. One of these scenarios resulted in an "insider" exercise with the overall objective to test the top management and contingency plans for their ability to respond to an insider threat. In this exercise, the top management of SVA was involved in handling an insider threat and a presumptive loss of a biological agent. The results from the "insider" exercise showed unambiguously that the participants found the exercise relevantly structured and well executed, and the overall objectives were achieved.

The results of the exercise clearly showed that it was necessary and valuable to involve top management in this kind of exercise and that disease investigation and crime investigation in parallel challenge the organization. Furthermore, it showed that joint training between SVA and the police increased knowledge and understanding of each other's competence and way of working. Taken together it can be concluded that the results of the exercise contributed to the improvement and revision of the contingency plans at SVA.

\section{Discussion}

\section{Gap Analysis}

The 5 participating institutes comply with current legislation and standards/recommendations for biosafety and biosecurity in their respective countries. In the framework of the
AniBioThreat project, the institutes performed an inventory to analyze to what extent, at the current initial phase of implementation, they comply with CWA 15793 and to identify areas where improvements can be made. The overall results of the inventory showed that written procedures were established for three-quarters of the (sub)items. However, only about half of these procedures fulfilled the requirements for compliance. The noncompliance was, for example, identified for consultation and communication (section 4.4.3), checking and corrective actions (section 4.5), and review (section 4.6). These findings are in accordance with the results presented by the Emory institute regarding compliance with CWA $15793 .{ }^{24}$ Checking and corrective actions as well as review were the weakest points at the Emory institute. ${ }^{24}$ It is important to emphasize that the gap analysis gave information about areas where compliance to CWA 15793 existed or not and thus gave information on which areas should be prioritized in the further implementation process. However, more in-depth analysis is needed to be able to make detailed conclusions about these areas.

From these results it can be concluded that at the 5 partner institutes, not only are efforts needed to establish procedures for (sub)items that are not yet covered, but also that efforts are required to reach compliance with already existing procedures. To enhance the level of compliance with existing procedures, training and education of staff and top management, as well as raising awareness of the contents of CWA 15793 is important. To promote the implementation process, the PDCA cycle, which is an essential part of CWA 15793, must be used, since it is a valuable tool for checking, for instance, the level of compliance with existing procedures by audits and inspections. This is consistent with other management systems (eg, EN ISO 9001:2008, ${ }^{7}$ EN ISO $14001: 2004,{ }^{8}$ OHSAS 18001:2007 ) but is absent in current legislation for working safely with biological agents.

\section{Biosafety and Biosecurity}

Biosecurity in this article refers to the principles, technologies, and practices that are implemented to secure pathogens, toxins, and sensitive technologies from unauthorized access, loss, theft, misuse, diversion, or intentional release. ${ }^{4}$ Within the existing procedures of the participating institutes, some biosecurity areas seemed to be less well represented compared to biosafety. Whereas, for example, all institutes have a worker health program to protect personnel from detrimental health effects caused by workplace exposure to biological agents and toxins, several institutes have not developed a clear policy regarding personal security support or training. This difference probably has to do with the fact that biosafety has a longer history in several national and international regulations, while biosecurity received less attention until the attack on the World Trade Center in 2001 and the subsequent anthrax letter mailings. It is noteworthy that several routines and topics are shared between the 2 disciplines and have historically, to a great 
extent, been covered by biosafety policies (Table 1). However, the observation that biosecurity procedures are not as well implemented or seen as a biosafety procedure makes clear that additional attention is needed for certain biosecurity areas. In that respect, CWA 15793 is a useful tool to improve in a structured way biosecurity issues.

The different international and national directives and regulations discussed above show that the 5 partner institutes not only have to deal with quite a number of different biosafety and biosecurity regulations but also that these regulations and guidelines often have differing perspectives and cover different aspects of biosafety and biosecurity. Furthermore, differences in the implementation of European directives for biosafety by EU member states in their own national laws as well as national biosafety guidelines also lead to ambiguities. These remarks underline the need for harmonization of biosafety and biosecurity, and, for the purpose of being able to work together in an LRN, the implementation of a management system and performance measurement tool such as the CWA 15793 is necessary.

\section{The Next Frontier for CWA 15793}

The CWA 15793 management system can assist in interlaboratory work, such as a European LRN, by helping to ensure that work is performed in a structured way and with continuous improvements applying the PDCA principle. This is exemplified by the recent European LRN initiatives QUANDHIP $^{25}$ and ERINHA, ${ }^{26}$ which are implementing the CWA 15793 management system to aid integration.

Implementation of the CWA 15793 management system can facilitate interlaboratory work such as sending and receiving samples, sharing liability, and exchange of staff between institutes. Validation and certification of methods in laboratories participating in such a network is of major importance in order to increase performance and reliability of results in emergency and crisis situations.

DG Enterprise and Industry has written a note on further standardization activities regarding the concept of interoperability. ${ }^{27}$ In this document CWA 15793 is outlined as a relevant work. This emphasizes the increased interest in and need for a robust international management system to better control laboratory biorisks. In September 2014 CWA 15793 is due to expire and be withdrawn by CEN. However, an initiative is in progress to transform CWA 15793 into an appropriate ISO-deliverable or other internationally recognized standard. ${ }^{28}$

\section{Implementation of CWA 15793}

CWA 15793 can facilitate credibility by aiding integration, enabling the sending and receiving of samples, and establishing trust toward a future European LRN. The implementation of CWA 15793 is an assurance that work done at the laboratories is performed in a structured way with continuous improvements.
Future activities in the AniBioThreat project will focus on developing ideas and initiatives to establish an implementation plan. For implementation of CWA 15793, full support of the top management is needed as well as resources for operation and maintenance. In the AniBioThreat project, the conclusion so far is that the gap analysis is a good start and a very useful tool to get information about what is in place and where improvements should be made. The exercises are also an important tool to verify the implementation of plans, routines, and instructions and should be used regularly in various scenarios as a measurement of compliance.

The use of existing networks is essential in the implementation process, and both LRN and other networks in the biorisk field can be useful as discussion and learning partners. As an example, concerning external audits, the networks can act as interagency audits. This could also be an opportunity to exchange knowledge and experience.

The conclusions and observations from the insider emergency exercise support the results of the gap analysis: that scenarios, as a bridging tool, are useful and relevant for continued work. The development of exercises in AniBioThreat contributes to a technical source and inspiration for similar exercises with other partners and networks. Future activities in the AniBioThreat project should focus on developing ideas and initiatives to establish an implementation plan to harmonize the national LRNs in bioterrorism response. In summary, the following practical steps can provide valuable guidance and tools for further implementation of CWA 15793:

- Engagement and support from top management;

- Raising awareness and knowledge about CWA 15793; all employees involved in work with biological agents should be informed and involved in the implementation process;

- Development and use of a compliance checklist;

- Performance of gap analysis;

- Drafting and use of emergency scenario booklet;

- Regular training and exercises;

- Use of existing networks (like LRN and others), where the implementation process has been started;

- Interagency audits between institutes and external audits; and

- Promotion and support from policymakers.

\section{ACKNOWLeDgments}

Writing of this publication has been supported by grants from the Swedish Civil Contingencies Agency (Anslag 2:4 Krisberedskap)(SMI and SVA) and the framework of the EU project AniBioThreat (Grant Agreement: Home/2009/ ISEC/AG/191) with financial support from the Prevention of and Fight against Crime Programme of the European Union, European Commission-Directorate General Home Affairs. This publication reflects views only of the authors, and the European Commission cannot be held responsible for any use that may be made of the information contained therein. 


\section{REFERENCES}

1. Commission of the European Communities. Communication from the Commission to the European Parliament and the Council on Strengthening Chemical, Biological, Radiological and Nuclear Security in the European Union - an EU CBRN Action Plan. Brussels; 2009. http://ec.europa.eu/dgs/homeaffairs/what-we-do/policies/crisis-and-terrorism/securingdangerous-material/index_en.htm. Accessed July 9, 2013.

2. CEN Workshop Agreement. CWA 15793. Laboratory biorisk management. CEN website. September 2011. http:// www.cen.eu/CEN/sectors/technicalcommitteesworkshops/ workshops/Pages/ws31.aspx. Accessed July 9, 2013.

3. World Health Organization. Laboratory Biosafety Manual. 3rd ed. Geneva: World Health Organization; 2004. http:// www.who.int/csr/resources/publications/biosafety/Biosafety7. pdf. Accessed July 9, 2013.

4. World Health Organization. Biorisk Management: Laboratory Biosecurity Guidance. Geneva: World Health Organization; 2006. http://www.who.int/csr/resources/publications/biosafety/ WHO_CDS_EPR_2006_6.pdf. Accessed July 9, 2013.

5. Salerno RM, Gaudioso J. Laboratory Biosecurity Handbook. Boca Raton, FL: CRC Press; 2007.

6. Clevestig P. Handbook of Applied Biosecurity for Life Science Laboratories. Solna, Sweden: Stockholm International Peace Research Institute (SIPRI); 2009.

7. ISO 9001:2008. Quality management systems - Requirements. ISO website. http://www.iso.org/iso/catalogue_detail? csnumber $=46486$. Accessed July 9, 2013.

8. ISO 14001:2004. Environmental management systems Requirements with guidance for use. ISO website. http:// www.iso.org/iso/catalogue_detail?csnumber $=31807$. Accessed July 9, 2013.

9. OHSAS 18001:2007. Occupational health and safety. Occupational Health \& Safety Standards website. http://www. osha-bs8800-ohsas-18001-health-and-safety.com/. Accessed July 9, 2013.

10. Morse SA, Kellogg RB, Perry S, et al. Detecting biothreat agents: the laboratory response network. ASM News 2003; 9:433-437.

11. CRTI. Chemical, Biological, Radiological-Nuclear and Explosives Research and Technology Initiative. 2007 Annual Report. Canadian Research and Technology Initiative. 2007.

12. Australian Society for Infectious Diseases. Bioterrorism response advisory group [editorial]. Microbiology Australia 2004;25:46.

13. Hwang HS. The strategic plan for preparedness and response to bioterrorism in Korea.JPrev MedPublic Health2008;4:209-213.

14. Muribi M, Andersson I, Allard Bengtsson U, Lindström A, Lindal E. Kunskapsuppbyggnad kring transport av misstänkt smittförande prov från fält till säkerhetslaboratorier. 2011. Svenska Laboratorieresponsnätverket (LRN). SVA:s rapportserie 17 ISSN 1654-7098.

15. Landelijk Laboratorium Netwerk terreur aanslagen. RIVM homepage. 2013. http://www.rivm.nl/Onderwerpen/ Onderwerpen/O/Ongevallen_en_rampen/Terrorisme. Accessed July 9, 2013.

16. National Coordinator for Counterterrorism (NCTb). Countering CBRN terrorism and responding to disasters. 2005 progress report, 14 March 2006.

17. Binder P. French National Network of Biotox-Piratox laboratories: a network of integrated networks to address the need for analyses in the event of a terrorist threat. EuroReference No. 7, ER07-12RX01. 2012. http://www.ansespro.fr/euroreference/ numero7/index.htm. Accessed July 9, 2013.

18. Directorate-General Home Affairs, European Commission, Brussels, Belgium, AniBioThreat Grant Agreement Home/ 2009/ISEC/AG/191.

19. One Health Initiative will unite human and veterinary medicine. One Health Initiative website. http://www.onehealthinitiative. com/. Accessed July 9, 2013.

20. European directive 2000/54/EC - biological agents at work. European Agency for Safety and Health at Work website. https://osha.europa.eu/en/legislation/directives/exposure-tobiological-agents/77. Accessed July 9, 2013.

21. Directive 2009/41/EC of the European Parliament and of the Council of 6 May 2009 on the contained use of genetically modified micro-organisms. 2009. http://www.biosafety. be/PDF/2009_41_EN.pdf?REQUEST = Seek-Deliver \& COLLECTION $=$ oj\&SERVICE $=$ eurlex $\&$ LANGUAGE $=$ en $\&$ DOCID = 20011073p0032. Accessed July 9, 2013.

22. EuFMD. European Commission for the Control of Footand-Mouth Disease. Minimum standards for laboratories working with FMDV in vitro/in vivo. Appendix 10 of European Commission Decision 2009/869/EC. April 30, 2009. http://www.fao.org/ag/againfo/commissions/docs/genses38/ appendix_10.pdf. Accessed July 25, 2013.

23. CEN Workshop 55-Guidance Document for CWA 15793:2008 'Laboratory Biorisk Management Standard.' http://www.cen.eu/cen/Sectors/TechnicalCommitteesWork shops/Workshops/Pages/CWA15793-guide.aspx. Accessed July 25, 2013.

24. Kalpana R. Initiation of ABSL3 influenza work based on the Biorisk Management system (CWA 15793:2008). 2012 ABSA Annual Biological Safety Conference; October 2012; Orlando, FL.

25. QUANDHIP. Project: Quality Assurance Exercises and Networking on the Detection of Highly Infectious Pathogens. QUANDHIP website. http://www.quandhip.info/Quandhip/ EN/Home/Homepage_node.html. Accessed July 9, 2013.

26. ERINHA. European Research Infrastrucure on Highly Pathogenic Agents website. http://www.erinha.eu/news/news. html. Accessed July 9, 2013.

27. Note on future standardisation activities. DG ENTR-H4/ IM-S/D. European Commission, Directorate-General Enterprise and Industry, Brussels, Belgium, 2010. http://rp7.ffg. at/upload/medialibrary/Interoperability_Standardisation.pdf. Accessed July 25, 2013.

28. US Department of Energy and Sandia National Laboratories. Evolution of CEN Workshop Agreement 15793: 2011: An Evaluation of Options. February 2013.

Manuscript received January 29, 2013;

accepted for publication May 17, 2013.

Addsress correspondence to: Bo Sundqvist National Veterinary Institute (SVA) SE-751 89 Uppsala Sweden

E-mail: bo.sundqvist@sva.se 